\title{
A Review on various Approaches for Feature Extraction in CBIR
}

\author{
Damandeep Singh $^{1}$, Nitin Bhagat ${ }^{2}$ \\ ${ }^{1,2}$ Assistant Professor, Sri Sai College of Engineering and Technology, Amritsar, Punjab, India
}

\begin{abstract}
Content Base image retrieval system is branch that uses for retrieval of different images on the basis of contents. Contents may be various attributes available in the images. A number of algorithms had been used for the purpose of content base Image retrieval. In this paper various approaches has been discussed that can be used for content base Image retrieval system. In this paper color base, energy based, shape and texture features extraction approaches has been discussed. These different approaches can be used for development of content base image retrieval system.
\end{abstract}

Keywords: CBIR, SIFT, Histogram, and color moments.

\section{Introduction}

\subsection{Content-based image retrieval (CBIR),}

Content-based image retrieval (CBIR), also known as query by image content (QBIC) and content-based visual information retrieval (CBVIR) is the application of computer vision techniques to the image retrieval problem, that is, the problem of searching for digital images in large databases (see this survey for a recent scientific overview of the CBIR field). Content-based image retrieval is opposed to traditional concept-based approaches "Content-based" means that the search analyzes the contents of the image rather than the metadata such as keywords, tags, or descriptions associated with the image.

The term "content" in this context might refer to colors, shapes, textures, or any other information that can be derived from the image itself. CBIR is desirable because searches that rely purely on metadata are dependent on annotation quality and completeness.

Having humans manually annotate images by entering keywords or metadata in a large database can be time consuming and May not capture the keywords desired to describe the image. The evaluation of the effectiveness of keyword image search is subjective and has not been welldefined. In the same regard, CBIR systems have similar challenges in defining success

\section{2 techniques of CBIR}

\section{a) Query techniques}

Query by example is a query technique that involves providing the CBIR system with an example image that it will then base its search upon. The underlying search algorithms may vary depending on the application, but result images should all share common elements with the provided example.

Options for providing example images to the system include:

- A preexisting image may be supplied by the user or chosen from a random set.
- The user draws a rough approximation of the image they are looking for, for example with blobs of color or general shapes.

This query technique removes the difficulties that can arise when trying to describe images with words.

\section{b) Semantic retrieval}

Semanticretrieval starts with a user making a request like "find pictures of Abraham Lincoln". This type of openended task is very difficult for computers to perform Lincoln may not always be facing the camera or in the same pose. Many CBIR systems therefore generally make use of lower-level features like texture, color, and shape. These features are either used in combination with interfaces that allow easier input of the criteria or with databases that have already been trained to match features (such as faces, fingerprints, or shape matching). However, in general, image retrieval requires human feedback in order to identify higher-level concepts.

\section{c) Relevance Feedback (Human Interaction}

Combining CBIR search techniques available with the wide range of potential users and their intent can be a difficult task. An aspect of making CBIR successful relies entirely on the ability to understand the user intent. CBIR systems can make use of relevance feedback, where the user progressively refines the search results by marking images in the results as "relevant", "not relevant", or "neutral" to the search query, then repeating the search with the new information. Examples of this type of interface have been developed.

\section{Literature Survey}

Ponciano-Silva, et al [1] Đoes a CBIR system really impact decisions of physicians in a clinical environment?" Content-based image retrieval systems are employed in several areas. One of the most prominent areas is the medical field, due to the huge volume of digital images daily generated in healthcare institutions employed for decision making. There are several works applying CBIR techniques over medical images. However, the great majority of them do not verify whether the systems are actually considered by the specialists as a pontential aid in a real environment. In 


\section{International Journal of Science and Research (IJSR) \\ ISSN (Online): 2319-7064 \\ Index Copernicus Value (2013): 6.14 | Impact Factor (2014): 5.611}

order to fill this research void in the literature, this work explores user experiments in a CBIR system involving resident physicians and radiologists.

Borowski, M. et al [2] Structuring the visual content of digital libraries using CBIR systems" Author describe the benefits of CBIR (content-based image retrieval) systems for retrieval and organization of visual content in databases of digital libraries. Unlike text-based retrieval systems which work with manually annotated keywords as metadata, CBIR systems use automatically extracted numerical representations of perceptual features such as color, texture or shape. This technique enables users of digital libraries to retrieve visual content without the help of textual metadata, which in some cases may be either nonexistent or not sufficient for a special purpose. It is our approach to support maintainers of digital libraries in organizing large image volumes using CBIR systems. Finding clusters of similar content or providing clusters with suitable keywords are new application contexts of CBIR. We propose a method for structuring visual content and evaluate retrieval results of CBIR systems with regard to their applicability to this method.

Yihua Lan et al [3] Similarity in Mammography CAD Using CBIR Approach: A Validation Study" To provide assistance for radiologists in mammographic screening, many computer-aided detection and diagnosis systems (CAD) have been developed. However, there are a lot of problems which should be addressed in conventional mammographic CAD system, such as the relatively lower performance in detecting malignant masses, especially those subtle masses. The reasons which caused those errors may be the black-box type approach, which only cuing those suspicious masses but it is different to explain the reasoning of the CAD decision-making. Mammographic CAD using content-based image retrieval is another new type of CAD which can provide visual assistance instead of the type of black box method in conventional CAD for radiologists.

ShiXi Tang et al [4] Further Study on the Design of Multilanguage Web CBIR Searching Engine Based on MAS" To solve the searching problem of multi-language Web in Tibetan, Chinese and English based on CBIR, the scheme of multi-language CBIR searching engine platform based on MAS, the general architecture, the general plan of searching engine based on Tibetan CBIR and the plan of knowledge data-base of searching engine based on Tibetan CBIR are improved by further study of the former based on the key techniques of $\mathrm{CSCW}$ (computer supported cooperative work) and PC (pervasive computing). And the result of pilot study is given too.

Marcher aet al [5] - CBIR for medical images - an evaluation trial"Content-based image retrieval (CBIR) has the potential to provide medical doctors with a powerful resource to help make accurate diagnoses. To aid in diagnosis, a CBIR system must retrieve similar images from the same (unknown) disease class as the patient. We have implemented a CBIR system that first predicts the disease class of the query image and then retrieves the $\mathrm{n}$ images nearest to the query image from the pool of images with the predicted disease class. With the cooperation of residents/radiologists at Indiana University Medical Center and the Department of Radiology at the University of Wisconsin we have recently completed an evaluation of our system. The results show that when using our system, the diagnostic accuracy of the group increased on average by $32 \%$ over diagnosis without any reference materials.

Lv Hue et al [6] -ALong-Term Learning Algorithm in CBIR Based on Log-Analyzing" the amount of images increases drastically. Content-based image retrieval (CBIR) has been proposed to efficiently manage these images. Traditional CBIR system extracts the low features of image automatically. Because of the difference between human comprehension and machine, the search results provided by CBIR system always cannot satisfy the user's need. So relevance feedback has been used in content-based image retrieval (CBIR) to bridge the semantic gap, which is existing between image low-level features and high-level human perceptions. This paper proposes an extendedjudging algorithm to analyze the information which includes both positive relevance and negative relevance. It uses the feedback $\log$ data and index table to expand the set of relevant images, and judging images in database by the current feedback record. Results show that compared with the traditional long-term learning method, the retrieval performance can be improved apparently.

Ambika, P. et al [7] $\checkmark$ isual query exploration process and generation of voluminous Image database for CBIR Systems" Content Based Image Retrieval is an approach for retrieving semantically-relevant images from an image database based on automatically-derived image features. Image retrieval is the process of browsing, searching and retrieving images from a large database of digital images. The collection of images in the web are growing larger and becoming more diverse. Retrieving images from such large collections and constructing an image database for CBIR systems are challenging problem. In this paper we have addressed a functional way to construct database for CBIR systems using multi-paradigm functional programming language. Schematic Representation of the database structure for the Content-Based Image Retrieval (CBIR) system can also be discussed. Visual exploration system enables users to search, browse and explore multimedia databases in an interactive and playful manner. We propose a query evaluation scheme to reduce the total number of similarity computations.

\section{Approaches Used}

\section{SIFT (Scale Invariant Feature Transform)}

Scale Invariant Feature Transform (SIFT) is an image descriptor for image-based matching and recognition developed by David Lowe. This descriptor as well as related image descriptors is used for a large number of purposes in computer vision related to point matching between different views of a 3-D scene and view-based object recognition. The SIFT descriptor is invariant to translations, rotations and scaling transformations in the image domain and robust to moderate perspective transformations and illumination variations. Experimentally, the SIFT descriptor has been proven to be very useful in practice for image matching and object recognition under real-world conditions.

\section{Volume 5 Issue 1, January 2016}




\section{International Journal of Science and Research (IJSR) \\ ISSN (Online): 2319-7064}

Index Copernicus Value (2013): 6.14 | Impact Factor (2014): 5.611

\section{Histogram}

A histogram is a graphical representation of the distribution of numerical data. It is an estimate of the probability distribution of a continuous variable (quantitative variable) and was first introduced by Karl Pearson. To construct a histogram, the first step is to "bin" the range of values - that is, divide the entire range of values into a series of small intervals - and then count how many values fall into each interval. A rectangle is drawn with height proportional to the count and width equals to the bin size, so that rectangles about each other. A histogram may also be normalized displaying relative frequencies. It then shows the proportion of cases that fall into each of several categories, with the sum of the heights equaling 1 . The bins are usually specified as consecutive, non-overlapping intervals of a variable. The bins (intervals) must be adjacent, and usually equal size. ${ }^{[2]}$ The rectangles of a histogram are drawn so that they touch each other to indicate that the original variable is continuous.

\section{Energy}

Energy is a property of objects which can be transferred to other objects or converted into different forms, but cannot be created or destroyed. It is difficult to give a comprehensive definition of energy because of its many forms, but one common definition is that it is the ability of a system to perform work. In SI units, energy is measured in joules. All of the many forms of energy are convertible to other kinds of energy, and obey the conservation of energy. Common energy forms include the kinetic energy of a moving object, the radiant energy carried by light, the potential energy stored by an object's position in a force field,(gravitational, electric or magnetic) elastic energy stored by stretching solid objects, chemical energy released when a fuel burns, and the thermal energy due to an object's temperature.

\section{Color Moment}

Color moments are measures that characterize color distribution in an image in the same way that central moments uniquely describe a probability distribution. Color moments are mainly used for color indexing purposes as features in image retrieval applications in order to compare how similar two images are based on color. Usually one image is compared to a database of digital images with precomputed features in order to find and retrieve a similar imag

\section{Conclusion}

Content base image retrieval system provides different images from the group of images on the basis of different relevant contents. In the content based image retrieval system different attributes of the image has been used for extraction of relevant images. In this paper different algorithms has been discussed that has been used for features extraction from an image. Scale invariant feature transformation approach has been used for extraction of different color images features. Histogram features that provides information about different intensity levels available in the image. Color features extracted by using color moment features. On the basis of study of different algorithm one can conclude that SIFT algorithm provide best features for different color images than other approaches. This approach is better in terms of computation complexity and computational cost.

\section{References}

[1] Ponciano-Silva, Đoes a CBIR system really impact decisions of physicians in a clinical environment?" IEEE Conf. on Computer-Based Medical Systems (CBMS), 2013, pp $41-46$.

[2] Borowski, M. Structuring the visual content of digital libraries using CBIR systems" IEEE Conf. on Information Visualization, 2000, pp 288 - 293.

[3] Yihua Lan Similarity in Mammography CAD Using CBIR Approach: A Validation Study" IEEE Conf. on Intelligent Human-Machine Systems and Cybernetics (IHMSC), 2012, pp $373-376$.

[4] ShiXi Tang Further Study on the Design of Multilanguage Web CBIR Searching Engine Based on MAS", IEEE Conf. on Pervasive Computing and Applications, 2006, pp $658-662$.

[5] Marchiori,A - CBIR for medical images - an evaluation trial" IEEE Conf. on Content-Based Access of Image and Video Libraries, 2001, pp 89 - 93.

[6] Lv Hue -ALong-Term Learning Algorithm in CBIR Based on Log-Analyzing" IEEE Conf. on Management and Service Science, 2009, pp 1 - 4.

[7] Ambika, P. $\forall$ isual query exploration process and generation of voluminous Image database for CBIR Systems" IEEE Conf. on Computing Communication \& Networking Technologies (ICCCNT), 2012, pp $1-7$. 